doi:10.13108/2013-5-3-3

UDC 517.53

\title{
DIRICHLET SERIES WITH REAL COEFFICIENTS
}

\author{
N.N. AITKUZHINA, A.M. GAISIN
}

\begin{abstract}
We study the class of entire functions represented by Dirichlet series with real coefficients determined by a convex growth majorant. We prove the criterion for the validity of an asymptotic identity on the positive ray which is an exact estimate for the growth of the logarithm of the modulus for each function in the considered class.
\end{abstract}

Keywords: Dirichlet series with real coefficients, discrete growth majorant.

Mathematics Subject Classification: 30D10.

\section{INTRODUCTION}

Let

$$
f(z)=\sum_{k=0}^{\infty} a_{k} z^{k} \quad(z=x+i y)
$$

be an entire transcendental function with real coefficients, and $\left\{p_{n}\right\}(n \geq 1)$ be the sequence of sign changes (by definition, $p_{n}=\min _{k>p_{n-1}}\left\{k: a_{p_{n-1}} a_{k}<0\right\}$, where $p_{0}=\min \left\{k: a_{k} \neq 0\right\}$ ). By $p(t)$ we denote the counting function of sequence $\left\{p_{n}\right\}, p(t)=\sum_{p_{n} \leqslant t} 1$. It was shown in [1] that if the density of the sequence $\left\{p_{n}\right\} \Delta=\lim _{t \rightarrow \infty} \frac{p(t)}{t}$ is equal to zero, then in each angle $\{z:|\arg z| \leqslant \varepsilon\}$ $(\varepsilon>0)$ entire function (1) has the same order as in the whole plane. Later it was found out that this result holds true also for the ray $\{z: \arg z=0\}$; if function (1) has a finite order $\rho$ and $\Delta=0$, then $[2]$

$$
\varlimsup_{x \rightarrow+\infty} \frac{\ln |f(x)|}{\ln M_{f}(x)}=1, \quad M_{f}(r)=\max _{|z|=r}|f(z)|(r>0) .
$$

It follows in particular that $\rho_{0}=\rho$, where $\rho_{0}=\varlimsup_{x \rightarrow+\infty} \frac{\ln \ln |f(x)|}{\ln x}$. As $\Delta=0$, identity (2) is valid also for the function of finite lower order [3]. In [4] there were found the best possible conditions for function $p(t)$ (being weaker than the condition $\Delta=0$ ) under those for each function of finite order (finite lower order) defined by series (1) the asymptotic identity

$$
\ln M_{f}(x)=(1+o(1)) \ln |f(x)|
$$

holds true as $x \rightarrow \infty$ outside some set of zero lower logarithmic density.

The aim of the present paper is to obtain similar with (3) asymptotic estimates for the entire functions defined by the Dirichlet series with real coefficients and having a more general growth majorant.

By $L$ we indicate the class of all continuous and increasing on $\mathbb{R}_{+}=[0,+\infty)$ positive functions. Let $\Phi \in L$ be a convex function such that its inverse $\varphi$ satisfies the condition

$$
\varlimsup_{x \rightarrow+\infty} \frac{\varphi\left(x^{2}\right)}{\varphi(x)}<\infty
$$

N.N. Aitkuzhina, A.M. Gaisin, Dirichlet series with Real Coefficients.

(c) Aitkuzhina N.N., Gaisin A.M. 2013.

The work is supported by RFBR (grant 12-01-97004-r_povolzhe_a).

Submitted April 15, 2013. 
By $A(\varphi)$ and $\underline{A}(\varphi)$ we denote the classes positive non-decaying on $\mathbb{R}_{+}$functions $\alpha=\alpha(t)$, $\alpha(t)=o(t \varphi(t))$ as $t \rightarrow \infty$, such that

$$
\varlimsup_{r \rightarrow \infty} \frac{1}{\varphi(r)} \int_{1}^{r} \frac{\alpha(t)}{t^{2}} d t=0, \quad \varliminf_{r \rightarrow \infty} \frac{1}{\varphi(r)} \int_{1}^{r} \frac{\alpha(t)}{t^{2}} d t=0,
$$

respectively. The subclasses $A(\varphi)$ and $\underline{A}(\varphi)$ consisting of the functions $\alpha \in L$ such that $\alpha(t) \geq \sqrt{t}$ are indicated by $W(\varphi)$ and $\underline{W}(\varphi)$, respectively.

Let $\Lambda=\left\{\lambda_{n}\right\}\left(0<\lambda_{n} \uparrow \infty\right)$ be a sequence satisfying the conditions

$$
\begin{aligned}
& \text { 1) } \sup _{t}(\lambda(t+1)-\lambda(t))<\infty \quad \text { (non-condentsabilty condition); } \\
& \text { 2) } \quad \ln \left(\lambda_{n+1}-\lambda_{n}\right)>-\alpha\left(\lambda_{n}\right) \quad(n \geq 1) \quad \text { (non-closing condition), }
\end{aligned}
$$

where $\alpha$ is a some function in $W(\varphi), \alpha(t)=O(t)$ as $t \rightarrow \infty, \lambda(t)=\sum_{\lambda_{n} \leqslant t} 1$. We denote by $D(\Lambda)$ the class of all entire functions $F$ represented by absolutely convergent in the whole plane Dirichlet series

$$
F(s)=\sum_{n=1}^{\infty} a_{n} e^{\lambda_{n} s} \quad(s=\sigma+i t)
$$

with real coefficients $a_{n}$. Let $M(\sigma)=\sup _{|t|<\infty}|F(\sigma+i t)|$,

$$
\underline{D}_{m}(\Phi)=\left\{F \in D(\Lambda): \exists\left\{\sigma_{n}\right\}, 0<\sigma_{n} \uparrow \infty, \ln M\left(\sigma_{n}\right) \leqslant \Phi\left(m \sigma_{n}\right)\right\} \quad(m \geq 1) .
$$

We let $\underline{D}(\Phi)=\bigcup_{m=1}^{\infty} \underline{D}_{m}(\Phi)$. By $\mu(\sigma)$ we indicate the maximal term of series $(6)$, i.e., $\mu(\sigma)=$ $\max _{n \geq 1}\left\{\left|a_{n}\right| e^{\lambda_{n} \sigma}\right\}$.

Let $\mu_{n}=\lambda_{p_{n}}$, where $\left\{p_{n}\right\}$ is the sequence of the sign changes for series $(6), l(t)=\sum_{\mu_{n} \leqslant t} 1$, $q(t)=\sum_{q_{n} \leqslant t} 1$, where

$$
q_{n}=\min \left(\frac{\lambda_{p_{n}}+\lambda_{p_{n+1}}}{2}, \lambda_{p_{n}}+1\right) .
$$

Since $\mu_{n}<q_{n}<\mu_{n+1}$, then $|l(t)-q(t)| \leqslant 1$.

Below we shall assume the following condition for sequence $\left\{p_{n}\right\}$ : there exists $\theta \in A(\varphi)$ such that

$$
\int_{1}^{\lambda_{n}} \frac{l\left(t ; \lambda_{n}\right)}{t} d t \leqslant \theta\left(\lambda_{n}\right) \quad(n \geq 1)
$$

where $l\left(t ; \lambda_{n}\right)$ is the number of the points $\mu_{j}$ in the segment $\left\{h:\left|h-\lambda_{n}\right| \leqslant t\right\}$. We note that in the case $\varphi(x)=\ln x$, condition (7) holds true immediately (it was shown in [4]).

In the present paper we prove the following theorem.

Theorem 1. Suppose condition (7). The asymptotic identity

$$
\ln M(\sigma)=(1+o(1)) \ln |F(\sigma)|,
$$

holds true as $\sigma \rightarrow \infty$ outside some set $E \subset[0, \infty)$ of zero lower density if and only if $l \in A(\varphi)$.

We note that if $\Phi(\sigma)=\underbrace{\exp \exp \ldots \exp }_{k}(\sigma)(k \geq 1)$, then as $k=1$, class $\underline{D}(\Phi)$ comprises

Dirichlet series of finite Ritt lower order. This is why the appropriate results in [3]-[4] are corollaries of Theorem 1. 


\section{Auxiliary STATEMENTS}

We shall make use of the following Borel-Nevanlinna type lemma.

Lemma 1 [5]. Let $\Phi \in L$ and inverse $\varphi$ for function $\Phi$ satisfies condition (4). Let also $u(\sigma)$ be a non-decaying positive and continuous on $[0, \infty)$ function and

$$
\lim _{\sigma \rightarrow \infty} u(\sigma)=\infty, \quad \underline{\lim _{\sigma \rightarrow \infty}} \frac{u(\sigma)}{\ln \Phi(\sigma)}<\infty .
$$

Let $\left\{x_{n}\right\}$ be a sequence chosen so that

$$
u\left(x_{n}\right) \leqslant C \ln \Phi\left(x_{n}\right), \quad 0<C<\infty .
$$

Suppose that a function $w$ belongs to class $W(\varphi)$. If $v=v(\sigma)$ solves equation

$$
w(v)=e^{u(\sigma)},
$$

then the asymptotic identity

$$
u\left(\sigma+d \frac{w(v(\sigma))}{v(\sigma)}\right)=u(\sigma)+o(1) \quad(0<d<\infty)
$$

holds true as $\sigma \rightarrow \infty$ outside some set $E \subset[0, \infty)$,

$$
\operatorname{mes}\left(E \cap\left[0, x_{n}\right]\right) \leqslant o\left(\varphi\left(v\left(x_{n}\right)\right)\right)+4 \int_{v\left(x_{1}\right)}^{v\left(x_{n}\right)} \frac{w^{*}(t)}{t^{2}} d t=o\left(\varphi\left(v\left(x_{n}\right)\right)\right), x_{n} \rightarrow \infty .
$$

In the hypothesis of Lemma $1, w^{*}$ is a some function being $w^{*}(t)=\beta(t) w(t), \beta \in L$. Function $\beta$ is chosen in such a way that $w^{*} \in W(\varphi)$. In Lemma 1 , the mentioned function $w^{*}$ always exists (it is not determined uniquely). We note that the exceptional set $E$ depends on function $w^{*}$.

In order to obtain an estimate like (8), we shall make use of the following statement on an estimate for a bounded analytic function in a circle.

Lemma 2. [6]. Let $g$ be a function analytic in the circle $\{z:|z|<R\}$, and

$$
|g(0)|>1, \quad \ln \sup _{|z|<R}|g(z)|=M<\infty .
$$

If $0<r<1-N^{-1}(N>1)$, there exists at most countable number of circles $V_{n}=\left\{z:\left|z-z_{n}\right|<\right.$ $\left.\rho_{n}\right\}$ such that

$$
\sum_{n} \rho_{n} \leqslant R r^{N}(1-r)
$$

and outside these circles but inside the circle $\{z:|z| \leqslant r R\}$ the estimate

$$
\ln |g(z)| \geq \frac{R-r}{R+r} \ln |g(0)|-5 N L
$$

holds true, where

$$
L=\frac{1}{2 \pi} \int_{0}^{2 \pi} \ln ^{+}\left|g\left(r e^{i \theta}\right)\right| d \theta-\ln |g(0)|
$$

Estimate (10) is more precise than the estimate $\ln |g(z)| \geq-5 N M$ in [7].

Let $\left\{p_{n}\right\}$ be a sequence of natural numbers, $\mu_{n}=\lambda_{p_{n}}, l(t)=\sum_{\mu_{n} \leqslant t} 1$,

$$
q_{n}=\min \left(\frac{\lambda_{p_{n}}+\lambda_{p_{n+1}}}{2}, \lambda_{p_{n}}+1\right), \quad q(t)=\sum_{q_{n} \leqslant t} 1 .
$$


We let

$$
Q_{a}(z)=\prod_{q_{n} \leqslant 2 a}\left(1-\frac{z^{2}}{q_{n}^{2}}\right) \quad\left(a \geq q_{1}\right)
$$

We have

Lemma 3 [4]. For each $\lambda_{n} \leqslant a\left(a \geq q_{1}\right)$ the estimate

$$
-\ln \left|Q_{a}\left(\lambda_{n}\right)\right| \leqslant \int_{0}^{\lambda_{n}} \frac{q\left(t ; \lambda_{n}\right)}{t} d t+4 N_{q}(2 e a)
$$

holds true, where $q\left(t ; \lambda_{n}\right)$ is number of points $q_{i}$ in the segment $\left\{h:\left|h-\lambda_{n}\right| \leqslant t\right\}$,

$$
N_{q}(t)=\int_{0}^{t} \frac{q(x)}{x} d x
$$

\section{Proof of Theorem 1}

1. Sufficiency. Since $|l(t)-q(t)| \leqslant 1, l \in A(\varphi)$, then $q \in A(\varphi)$. Next,

$$
\int_{0}^{r} \frac{q(t)}{t^{2}} d t=\int_{0}^{r} \frac{d N_{q}(t)}{t}=\frac{N_{q}(r)}{r}+\int_{0}^{r} \frac{N_{q}(t)}{t^{2}} d t
$$

where $N_{q}(t)=\int_{0}^{t} \frac{q(x)}{x} d x$. Therefore, $N_{q} \in A(\varphi)$. Hence, there exists a continuous on $[0, \infty)$ function $\beta_{1}(t), 1 \leqslant \beta_{1}(t) \uparrow \infty, t \rightarrow \infty$, such that the function $N_{q}(2 e t) \beta_{1}(t)$ also belongs to $A(\varphi)$.

Let us estimate the integral $\int_{0}^{\lambda_{n}} \frac{q\left(t ; \lambda_{n}\right)}{t} d t$, where $q\left(t ; \lambda_{n}\right)$ is the number of the points $q_{i}$ in the segment $\left\{h:\left|h-\lambda_{n}\right| \leqslant t\right\}$. Bearing in mind the latter of conditions (5), we have

$$
\int_{0}^{\lambda_{n}} \frac{q\left(t ; \lambda_{n}\right)}{t} d t=\int_{\gamma_{n}}^{1} \frac{q\left(t ; \lambda_{n}\right)}{t} d t+\int_{1}^{\lambda_{n}} \frac{q\left(t ; \lambda_{n}\right)}{t} d t=I_{1}+I_{2},
$$

where $\gamma_{n}=\frac{1}{2} e^{-\alpha\left(\lambda_{n}\right)}$. But it follows from the condition $\sup (\lambda(t+1)-\lambda(t))<\infty$ that $q\left(t ; \lambda_{n}\right) \leqslant$ $d t+d(0<d<\infty)$. Since $\mu_{n}<q_{n}<\mu_{n+1}$, then $\left|q\left(t ; \lambda_{n}\right)-l\left(t ; \lambda_{n}\right)\right| \leqslant 1$. Thus, taking into account (7), we have

$$
I_{1} \leqslant d\left[1+\ln 2+\alpha\left(\lambda_{n}\right)\right], \quad I_{2} \leqslant \theta\left(\lambda_{n}\right)+\ln \lambda_{n} \quad(n \geq 1),
$$

where $\alpha \in W(\varphi), \theta \in A(\varphi)$. Hence, due to (12), we get that

$$
\int_{0}^{\lambda_{n}} \frac{q\left(t ; \lambda_{n}\right)}{t} d t<\Theta_{1}\left(\lambda_{n}\right)
$$

where $\Theta_{1} \in W(\varphi)$. Hence, there exists a continuous on $[0, \infty)$ function $\beta_{2}(t), 1 \leqslant \beta_{2}(t) \uparrow \infty$ as $t \rightarrow \infty$ such that the function $\Theta_{1}(t) \beta_{2}(t)$ belongs to $W(\varphi)$. Let $w^{*}(t)=\beta(t) w(t)$, where $w(t)=\Theta_{1}(t)+N_{q}(2 e t), \beta(t)=\min \left(\beta_{1}(t), \beta_{2}(t)\right)$. It is clear that $w^{*} \in \underline{W}(\varphi)$.

Let $v=v(\sigma)$ be a solution to the equation

$$
w^{*}(v)=3 \ln \mu(\sigma),
$$


where $\mu(\sigma)$ is the maximal term of the series (6). We let

$$
F_{a}(s)=\sum_{\lambda_{n} \leqslant a} a_{n} e^{\lambda_{n} s}, \quad F_{a}^{*}(s)=\sum_{\lambda_{n} \leqslant a} a_{n} Q_{a}\left(\lambda_{n}\right) e^{\lambda_{n} s},
$$

where

$$
Q_{a}(z)=\prod_{q_{n} \leqslant 2 a}\left(1-\frac{z^{2}}{q_{n}^{2}}\right) .
$$

Since all $a_{n} Q_{a}\left(\lambda_{n}\right)\left(\lambda_{n} \leqslant a\right)$ are of the same sign, we can assume that $a_{n} Q_{a}\left(\lambda_{n}\right) \geq 0\left(\lambda_{n} \leqslant a\right)$. It is obvious that

$$
M_{a}^{*}(\sigma)=\sup _{|t|<\infty}\left|F_{a}^{*}(\sigma+i t)\right|=F_{a}^{*}(\sigma)
$$

and thus

$$
M_{a}^{*}(\sigma)=\frac{1}{2 \pi i} \int_{|t|=\delta} q_{a}(t) F_{a}(t+\sigma) d t
$$

where $q_{a}(t)$ is the function associated in the Borel sense with $Q_{a}(z), \delta=\frac{w_{1}(v(\sigma))}{v(\sigma)}, w_{1}(t)=$ $\beta^{1 / 2}(t) w(t)$. Taking into consideration (14) and proceeding as in [3], one can show that as $\sigma \rightarrow \infty$

$$
\delta \max _{|t|=\delta}\left|q_{v}(t)\right| \leqslant \delta \int_{0}^{\infty} M\left(Q_{v}, r\right) e^{-\delta r} d v \leqslant \mu^{o(1)}(\sigma),
$$

where $M\left(Q_{v}, r\right)=\max _{|z|=r}\left|Q_{v}(z)\right|$. Applying them Lemma 1, we get that as $\sigma \rightarrow \infty$, outside some set $E_{1} \in[0, \infty)$ of zero lower density $d E_{1}$

$$
\ln \mu\left(\sigma+4 \delta^{*}\right)=(1+o(1)) \ln \mu(\sigma), \quad \delta^{*}=\frac{w^{*}(v(\sigma))}{v(\sigma)},
$$

and according to the same lemma, as $\sigma_{n}^{*} \rightarrow \infty$,

$$
\frac{\operatorname{mes}\left(E_{1} \cap\left[0, \sigma_{n}^{*}\right]\right)}{\sigma_{n}^{*}}=o(1), \sigma_{n}^{*} \rightarrow \infty,
$$

where the sequence $\left\{\sigma_{n}^{*}\right\}$ is chosen by the restriction $\ln M\left(\sigma_{n}^{*}\right) \leqslant \Phi\left(m \sigma_{n}^{*}\right)(m \geq 1)$. Then as $\sigma \rightarrow \infty$, outside $E_{1}$,

$$
\begin{gathered}
\sum_{\lambda_{n}>v(\sigma)}\left|a_{n}\right| e^{\lambda_{n}\left(\sigma+3 \delta^{*}\right)} \leqslant \mu\left(\sigma+4 \delta^{*}\right) \sum_{\lambda_{n}>v(\sigma)} e^{-\delta^{*} \lambda_{n}} \leqslant \\
\mu^{1+o(1)}(\sigma) \exp [-3(1+o(1)) \ln \mu(\sigma)]<1 .
\end{gathered}
$$

Taking into account the former of conditions (5), we see that $\lambda(v(\sigma))=O(v(\sigma))$ as $\sigma \rightarrow \infty$. Hence, $\ln \lambda(v(\sigma)) \leqslant 2 \ln v(\sigma) \leqslant 2 w(v(\sigma))$ as $\sigma \geq \sigma_{0}$ (we have used that $w \in W(\varphi)$ ). In view of (14), (17), as $\sigma \rightarrow \infty$, outside $E_{1}$ we have

$$
M\left(\sigma+3 \delta^{*}\right) \leqslant \mu\left(\sigma+4 \delta^{*}\right)\left[\lambda((v(\sigma))+1] \leqslant M^{1+o(1)}(\sigma), \quad \lambda(t)=\sum_{\lambda_{n} \leqslant t} 1 .\right.
$$

Therefore, as $\sigma \rightarrow \infty$, outside $E_{1}$,

$$
\ln M\left(\sigma+3 \delta^{*}\right)=(1+o(1)) \ln M(\sigma) .
$$

Taking into account (16), (18), by (15) we obtain that, as $\sigma \rightarrow \infty$, outside $E_{1}$,

$$
M_{v}^{*}(\sigma) \leqslant M^{1+o(1)}(\sigma)\left(\max _{|\xi-\sigma| \leqslant \delta}|F(\xi)+1|\right) .
$$


But as $\sigma \rightarrow \infty$, outside $E_{1}$,

$$
M(\sigma) \leqslant \sum_{\lambda_{n} \leqslant v(\sigma)}\left|a_{n}\right| e^{\lambda_{n} \sigma}+1=\sum_{\lambda_{n} \leqslant v(\sigma)}\left(\left|a_{n}\right|\left|Q_{v}\left(\lambda_{n}\right)\right| e^{\lambda_{n} \sigma}\right)\left|Q_{v}\left(\lambda_{n}\right)\right|^{-1}+1 .
$$

Together with Lemma 3, estimate (13), identity (14) it implies that, as $\sigma \rightarrow \infty$, outside $E_{1}$,

$$
M(\sigma) \leqslant \mu^{o(1)}(\sigma) M_{v}^{*}(\sigma) \leqslant M^{o(1)}(\sigma) M_{v}^{*}(\sigma) .
$$

In view of this estimate, it finally follows (20) that, as $\sigma \rightarrow \infty$, outside $E_{1}, d E_{1}=0$,

$$
M^{1+o(1)}(\sigma) \leqslant \max _{|\xi-\sigma| \leqslant \delta}|F(\xi)|=\left|F\left(\xi^{*}\right)\right|,
$$

where $\left|\xi^{*}-\sigma\right|=\delta, \delta=\frac{w_{1}(v(\sigma))}{v(\sigma)}, w_{1}(t)=\beta^{1 / 2}(t) w(t)$. In addition, according to Lemma 1,

$$
\frac{\operatorname{mes}\left(E_{1} \cap\left[0, \sigma_{n}^{*}\right]\right)}{\sigma_{n}^{*}}=o(1), \sigma_{n}^{*} \rightarrow \infty .
$$

We recall that sequence $\left\{\sigma_{n}^{*}\right\}$ is determined by the restriction $\ln M\left(\sigma_{n}^{*}\right) \leqslant \Phi\left(m \sigma_{n}^{*}\right)(m \geq 1)$ and $\sigma_{n}^{*} \rightarrow \infty$.

We let $B=[0, \infty) \backslash E_{1}$. There exists a sequence $\left\{\sigma_{i}\right\}\left(\sigma_{i} \in B\right)$ such that $\sigma \uparrow \infty, \sigma_{i}+\delta_{i} \leqslant \sigma_{i+1}$ and $\sigma_{i+1}-\delta_{i+1}<\inf \left\{\sigma: \sigma \in B, \sigma>\sigma_{i}+\delta_{i}\right\}$, where $\delta_{i}=\delta\left(v\left(\sigma_{i}\right)\right)(i \geq 1)$. Hence,

$$
B \subset \bigcup_{i=1}^{\infty}\left[\sigma_{i}-\delta_{i}, \sigma_{i}+\delta_{i}\right]
$$

We let $g(z)=F\left(z+\xi^{*}\right)$. It follows from (21) that $|g(0)|>1$ as $\sigma \in B \cap\left[\sigma_{0}, \infty\right)\left(\sigma_{0}>0\right)$. In (21) we let $\sigma=\sigma_{i}, \delta=\delta_{i}$, and in Lemma 2 we let $N=3, r=\left[\beta\left(v\left(\sigma_{i}\right)\right)\right]^{-1 / 2}, R=2 \delta_{i}^{*}$, $\left(\delta_{i}^{*}=\frac{w^{*}\left(v\left(\sigma_{i}\right)\right)}{v\left(\sigma_{i}\right)}\right)$. Then $R r=2 \frac{w^{*}\left(v_{i}\right)}{v_{i} \sqrt{\beta\left(v_{i}\right)}}=2 \sqrt{\beta\left(v_{i}\right)} \frac{w\left(v_{i}\right)}{v_{i}}=\frac{2 w_{1}\left(v_{i}\right)}{v_{i}}=2 \delta_{i}\left(v_{i}=v\left(\sigma_{i}\right)\right)$. Therefore, by Lemma 2 we conclude that in the circle $\left\{z:|z| \leqslant 2 \delta_{i}\right\}$ but outside exceptional circles $V_{n}^{(i)}$ with the total sum of radii satisfying the estimate

$$
\sum_{n} p_{n}^{(i)} \leqslant 2 \delta_{i} \beta_{i}^{-\frac{1}{2}}
$$

we have estimate $(10)$. Here $\beta_{i}=\beta\left(v\left(\sigma_{i}\right)\right)$. Then for each $z$ in the circle $\left\{z:|z| \leqslant \delta_{i}\right\}$ but outside the circles $V_{n}^{(i)}$ with the total sum of radii satisfying estimate $(22)$, as $i \rightarrow \infty$, by (10) we have

$$
\ln |g(z)| \geq\left[1+o(1)-15 \frac{L}{\ln |g(0)|}\right] \ln |g(0)| .
$$

Taking into consideration that $g(z)=F\left(z+\xi^{*}\right)$ and employing estimates (21), (19), (23), we obtain that for each $z$ in the circle $\left\{z:\left|z-\sigma_{i}\right| \leqslant \delta_{i}\right\}$ but outside exceptional circles $C_{n}^{(i)}$ with total sum of radii at most $2 \delta_{i} \beta_{i}^{-\frac{1}{2}}$,

$$
\ln |F(z)|>(1+o(1)) \ln M\left(\sigma_{i}\right), i \rightarrow \infty .
$$

Here $\delta_{i}=\delta\left(v\left(\sigma_{i}\right)\right)$, and $\delta=\frac{w(v(\sigma))}{v(\sigma)}, w(t)$ is the function satisfying the hypothesis of Lemma 1 .

Let $E_{2}$ be the projection of the set $\bigcup_{i, n} C_{n}^{(i)}$ on the real axis. Let us estimate the relative measure of set $E_{2}$. We choose a sequence $\left\{\sigma_{n}\right\}\left(\sigma_{n} \in B, B=[0, \infty) \backslash E_{1}\right)$ such that if $\sigma_{n}^{\prime}=$ $\inf \left\{\sigma: \sigma \in B, \sigma>\sigma_{n}+\delta_{n}\right\}$, then $0 \leqslant \sigma_{n+1}-\sigma_{n}^{\prime} \leqslant \delta_{n}$.

Let $\sigma_{k}<\sigma_{n}^{*} \leqslant \sigma_{k+1}$. Since $\ln M\left(\sigma_{n}^{*}\right) \leqslant \Phi\left(m \sigma_{n}^{*}\right)(m \geq 1)$, in view of (4), (14) we obtain that $\sigma_{n}^{*} \geq \alpha \varphi\left(v\left(\sigma_{n}^{*}\right)\right)>\alpha \varphi\left(v\left(\sigma_{k}\right)\right)(\alpha>0)$. Thus,

$$
\frac{\operatorname{mes}\left(E_{2} \cap\left[0, \sigma_{n}^{*}\right]\right)}{\sigma_{n}^{*}} \leqslant \frac{4}{\sigma_{k}} \sum_{i=1}^{k-1} \beta_{i}^{-\frac{1}{2}} \delta_{i}+\frac{4 \delta_{k}}{\alpha \varphi\left(v\left(\sigma_{k}\right)\right)},
$$


where

$$
\delta_{k}=\frac{\beta^{-\frac{1}{2}}\left(v\left(\sigma_{k}\right)\right) w\left(v\left(\sigma_{k}\right)\right)}{v\left(\sigma_{k}\right)}<\frac{w^{*}\left(v\left(\sigma_{k}\right)\right)}{v\left(\sigma_{k}\right)},
$$

and $w^{*}=\beta(t) w(t)(0<\beta(t) \uparrow \infty, t \rightarrow \infty)$ is a function satisfying the hypothesis of Lemma 1 . Therefore,

$$
\lim _{k \rightarrow \infty} \frac{4 \delta_{k}}{\alpha \varphi\left(v\left(\sigma_{k}\right)\right)}=0
$$

But since $\beta_{i}^{-1 / 2} \rightarrow 0$ as $i \rightarrow \infty$, and $\sigma_{k} \geq 2 \sum_{i=1}^{k-1} \delta_{i}$, then $d E_{2}=0$. Thus, estimate (8) holds true as $\sigma \rightarrow \infty$ outside $E=E_{1} \cup E_{2}, d E=0$. The proof of the sufficiency is complete.

2. Necessity. Let $l \notin A(\varphi)$. Then

$$
\varlimsup_{r \rightarrow \infty} \frac{1}{\varphi(r)} \sum_{\mu_{n} \leqslant r} \frac{1}{\mu_{n}}=\gamma>0
$$

and the series $\sum_{n} \mu_{n}^{-1}$ diverges. We let

$$
\Phi(z)=\prod_{n=1}^{\infty}\left(1-\frac{z^{2}}{\mu_{n}^{2}}\right) .
$$

Since $\mu_{n}=\lambda_{p_{n}}$, and sequence $\left\{\lambda_{n}\right\}$ obeys conditions (5), sequence $\left\{\mu_{n}\right\}$ has a finite upper density and $\mu\left(t ; \mu_{n}\right) \leqslant C t+C(0<C<\infty)$, where $\mu\left(t ; \mu_{n}\right)$ is the number of the points $\mu_{i}$ $\left(\mu_{i} \neq \mu_{n}\right)$ in the segment $\left\{h:\left|h-\mu_{n}\right| \leqslant t\right\}$. Hence, the results of paper [8] yield

$$
\ln \left|\Phi^{\prime}\left(\mu_{n}\right)\right|=-\int_{0}^{1} \frac{\mu\left(t ; \mu_{n}\right)}{t} d t+O\left(\mu_{n}\right) .
$$

Due to the latter condition in (5), it implies

$$
-\ln \left|\Phi^{\prime}\left(\mu_{n}\right)\right|=O\left(\mu_{n}\right), n \rightarrow \infty .
$$

We introduce the series

$$
F(s)=\sum_{k=1}^{\infty} a_{k} e^{\lambda_{n} s}(s=\sigma+i t)
$$

where

$$
a_{k}=\left\{\begin{array}{ll}
\frac{\psi^{2}\left(\mu_{n}\right)}{\Phi^{\prime}\left(\mu_{n}\right)\left(1+\mu_{n}\right)^{2}}, & k=p_{n}, \\
0, & k \neq p_{n}
\end{array} \quad(n \geq 1), \quad \psi(z)=\prod_{n=1}^{\infty}\left(1+z / \mu_{n}\right) e^{-z / \mu_{n}} .\right.
$$

Since $l(x)=O(x)$ as $x \rightarrow \infty$, bearing in mind (25), we have (cf. [8])

$$
\ln \psi(x) \leqslant-d x \varphi(x) \quad(x>0)
$$

where $0<d<\infty$. One can make sure that for sufficiently small $q>0$ the estimate

$$
\ln \psi^{2}(x) \leqslant-2 q x \sum_{\mu_{n} \leqslant x} \frac{1}{\mu_{n}} \quad(0<q<\infty)
$$

holds true. Therefore, in view of (26), (29), series (27) diverges absolutely in the whole plane. Since $|F(\sigma)|=O(1)$ as $\sigma \rightarrow+\infty[6]$, it is sufficient to show that $F \in \underline{D}(\Phi)$.

Due to (26), (29), for some $A \geq A_{0}$ we have

$$
M(\sigma) \leqslant A \exp \left[\max _{t \geq 0}\left(-q t \int_{0}^{t} \frac{l(x)}{x^{2}} d x+\sigma t\right)\right] .
$$


It follows from (25) that for some sequence $\left\{t_{k}\right\}, t_{k} \uparrow \infty$,

$$
\int_{0}^{t_{k}} \frac{l(x)}{x^{2}} d x \geq \beta \varphi\left(t_{k}\right), \quad \beta>0 .
$$

Let $t=t(\sigma)$ be a solution to the equation

$$
a \int_{0}^{t} \frac{l(x)}{x^{2}} d x=\sigma .
$$

Since it is obvious that $t=t(\sigma)$ is a continuous increasing function, by (30)-(32) we get that for some sequence $\left\{\sigma_{k}\right\}, \sigma_{k} \uparrow \infty$,

$$
\begin{gathered}
\ln M\left(\sigma_{k}\right) \leqslant \ln A+\sigma_{k} t_{k} \leqslant \ln A+\sigma_{k} \Phi\left(\frac{\sigma_{k}}{\beta q}\right) \leqslant \\
\leqslant \ln A+\Phi\left(\sigma_{k}\right) \Phi\left(\frac{\sigma_{k}}{\beta q}\right) \leqslant \ln A+\Phi^{2}\left(B \sigma_{k}\right) \quad(0<B<\infty) .
\end{gathered}
$$

Thus, $\ln M\left(\sigma_{k}\right) \leqslant \Phi\left(m \sigma_{k}\right), m \in \mathbb{N}, m \geq m_{0}$. Therefore, $F \in \underline{D}(\Phi)$. The proof of the necessity is complete.

\section{BIBLIOGRAPHY}

1. G. Pólya. Untersuchungen über Lücken und Singularitäten von Potenzeihen// Math. Z. 1929. V. 29. P. 549-640.

2. M.N. Sheremeta. A theorem of Polya // Ukrain. matem. zhur. 1983. V. 35, No. 1. P. 119-124. [Ukrainian Math. J. 1983. V. 35, No. 1. P. 108-112.]

3. M.N. Sheremeta. Entire functions with real Taylor coefficients // Ukrain. matem. zhur. 1985. V. 37, No. 6. P. 786-787. [Ukrainian Math. J. 1985. V. 37, No. 6. P. 647-648.]

4. A.M. Gaisin. On a theorem of Pólya on entire functions with real Taylor coefficients // Sib. matem. zhur. 1997. V. 38, No. 1. P. 46-55. [Siber. math. j. 1997. V. 38, No. 1. P. 37-46.]

5. N.N. Yusupova. Borel-Nevanlinna type theorem for function of given growth// Proceedings of XLIV International scientific student conference "Student and scientific and technical advance". Mathematics. Novosibirski. Novosibirsk state univ. 2006. P. 32 (in Russian).

6. A.M. Gaisin. On a conjecture of Pólya// Izv. RAN. Ser. matem. 1994. V. 58, No. 2. P. 73-92. [Russ. Acad. Sci. Izv. Math. 1995. V. 44, No. 2. P. 281-299.]

7. N.V. Govorov. On lower estimate for function subharmonic in circle // Teoriya funktsij, funktsionalnyj analiz i ih prilozheniya. 1968. No. 6. P. 130-150 (in Russian).

8. I.F. Krasichkov. Lower estimate for entire functions of finite order // Sibir. matem. zhur. 1965. V. 6, No. 4. P. 840-861 (in Russian).

Narkes Nurmukhametovna Aitkuzhina,

Bashkir State University,

Z. Validi str., 32, newline 450074, Ufa, Russia

E-mail: YusupovaN@rambler.ru

Akhtyar Magazovich Gaisin, Institute of Mathematics USC RAS,

Chernyshevsky str, 112,

450008, Ufa, Russia

E-mail: GaisinAM@mail.ru 